\title{
Speed of Gravitation and Electromagnetic Waves through the Dark Energy of Intergalactic Space and Its Implications of a Unified Theory
}

\author{
Frank R. Tangherlini \\ San Diego, CA, USA \\ Email: frtan96@gmail.com
}

How to cite this paper: Tangherlini, F.R. (2018) Speed of Gravitation and Electromagnetic Waves through the Dark Energy of Intergalactic Space and Its Implications of a Unified Theory. Journal of Modern Physics, 9, 573-583.

https://doi.org/10.4236/jmp.2018.94039

Received: February 13, 2018

Accepted: March 16, 2018

Published: March 19, 2018

Copyright $\odot 2018$ by author and Scientific Research Publishing Inc. This work is licensed under the Creative Commons Attribution International License (CC BY 4.0).

http://creativecommons.org/licenses/by/4.0/

\begin{abstract}
The recent finding that gravitational waves (GW170817) traveled at the same speed as electromagnetic (EM) waves (GRB 170817A) from a binary neutron star merger does not necessarily mean that they traveled throughout their journey at speed $c$. Some recent works by the author (2015) Journal of Modern Physics, 6, 78-87, and 1360-1370; (2016), 7, 1829-1844; (2017), 8, 622-635 show that the diminished brightness of Type Ia supernovae (SNe Ia) can be explained by assuming that dark energy, instead of having a negative pressure, has an index of refraction $n$, causing the speed of light through intergalactic space (IGS) to be reduced to $c / n$, with $n \approx 1.5$. It follows that GWs (not considered in the previous works) would also travel with speed $c / n$ through IGS. However, speed of GWs and EMWs within galaxies themselves is $c$. A brief review of the model is given, together with other predictions, e.g., new values for the Hubble constant and age of the universe, and necessary absence of correlation of neutrinos with gamma ray bursts (GRBs), in agreement with numerous searches. In the previous works, there were implications of a unified theory. If the model holds, since GWs would experience the same speed reduction as EMWs, this would further support unification. An improved falsification methodology for a previously proposed astronomical test based on discordant redshifts is given.
\end{abstract}

\section{Keywords}

Speed of Gravitational Waves, Dark Energy, Hubble Constant, Absence of Neutrino Correlation with GRBs

\section{Introduction}

The recent discovery that a gravitational wave GW170817, that resulted from a 
binary neutron star merger [1] [2] arrived at the same time (to within $\sim 1.7 \mathrm{~s}$ ) as the gamma ray burst GRB170817A [3] [4], and that electromagnetic (EM) radiation over a wide spectrum was detected within a short time period, as summarized in [1], demonstrated that gravitational waves (GWs) travel at the same speed as EMWs through intergalactic space (IGS) and the Milky Way (MW) with negligible dispersion. However, the purpose of this work is to point out that it does not necessarily follow that the GWs and EMWs traveled through the IGS with the vacuum speed of light, because of a new description of dark energy proposed by the author recently [5] [6] [7] [8], in which an alternative to the accelerating universe was introduced to provide another explanation for the increased apparent magnitude of the Type Ia supernovae ( $\mathrm{SNe}$ Ia) that led to the accelerating universe [9] [10] [11]. The model assumes that dark energy is only in IGS, and that it does not have a negative pressure causing the universe to accelerate, but for $z \leq 1$, later reduced to $z \leq 0.6$, it has an index of refraction $n$, with $n \approx 1.5$ that results in the speed of light through IGS to be reduced to $\sim 2 c / 3$. However, the speed of EMWs as well as GWs within the galaxies themselves is $c$. Although the particle nature of dark energy is left open, it is proposed that dark energy is a different phase of dark matter. The Einstein de Sitter (EdS) universe, with a multiplicative correction for distance, was used as the alternative to the $\Lambda \mathrm{CDM}$ universe, with which it was compared, in order to determine $n$ by a least squares fit which yielded $n \approx 1.5$. In the previous work [5] [6] [7] [8], there was no discussion of GWs, but now, because of the above finding that GWs travel at the same speed as EMWs, it follows that the model predicts that GWs also travel through IGS with reduced speed $c / n$. As a further prediction, it is shown that the reduced speed of light leads to a redetermination of the Hubble constant, which in turn leads to a resolution of a long-standing difficulty concerning the age of the EdS universe. Since no dispersion was observed over the optical range in the SNe Ia studies [9] [10] [11], it was noted in [6] that if this held over the entire EM spectrum, as has now been shown to be the case, this would provide a simple explanation for the absence of neutrino correlation with GRBs found by IceCube [12] [13], which is further extended in this work to include the negative findings of Borexino [14] [15]. Additional references to other negative findings are also given below. It is proposed that the predicted speed reduction through IGS for GWs that is the same as that for EMWs, together with a possible relation of electromagnetic parameters to gravitational parameters noted previously [6] [7] [8], as well as the absence of dispersion, are implications of a unified theory of gravitation and electromagnetism. There is a brief description, based on earlier work [8], on how the model can be tested astronomically by searching for heretofore ignored possible galactic sources of discordant redshifts, together with an improved discussion over that given previously of how this type of search could be used to falsify the model.

\section{Determination of Index of Refraction}

A least squares fit of $n$, denoted by $n(I s)$, to the increased apparent magnitude of 
the SNe Ia given in [16] for the $\Lambda \mathrm{CDM}$ universe, based on a comparison with the EdS universe, and a correction to the apparent magnitude $\delta m$, led to $n(l s)=1.49 \pm 0.04$ for $0.1 \leq z \leq 1.0$. The correction $\delta m$ was shown in [5] to be given by

$$
\delta m=5 \log (1+(n-1) \ln (1+z)),
$$

in which $n$ was taken to be a constant independent of $z$. Fiducial values of the cosmological density parameters, $\Omega_{m}=0.3, \Omega_{\Lambda}=0.7$, were used in [16]. In [5], Table 1 gives the percentage agreement for the increase in apparent magnitude of the SNe Ia for the EdS universe with that for the $\Lambda$ CDM universe, when the correction $\delta m$ is taken into account. The model can also account for the increased distances to the "standard ruler" of the baryon acoustic oscillations (BAO) [17] [18], with the logarithm of the fractional correction to the distance given by

$$
d=\log (1+(n-1) \ln (1+z)) .
$$

Also in [5], Table 3 makes the comparison for $d$. As expected, the two Tables are in perfect agreement when rounding-off errors are allowed for, since $\delta m=5 d$. The percentage of agreement of the model with $\Lambda \mathrm{CDM}$ ranges from $-6.7 \%$ for $z=0.1$, to $6.7 \%$ for $z=1.0$, with zero disagreement for $z=0.6$. For the $\mathrm{BAO}$, there are two distances: The angular distance $D_{A}$ and the luminosity distance $D_{L}$ that differ by factors of $(1+z)$. However, for the ratio $D_{A}(\Lambda \mathrm{CDM}) / D_{A}(\mathrm{EdS})$, and that for $D_{L}$, the $(1+z)$ factors cancel, so that they have the same logarithm given by $d$. This increase of distance, and hence size of the EdS universe, because of the reduced speed of light, can be shown to give an alternative explanation to the Large Scale Structure (LSS) studies that support the accelerating universe, but for brevity will not be given here.

\section{Dark Matter and Dark Energy}

It was also assumed that dark energy, characterized by its macroscopically-large index of refraction, results from the dark matter that was originally in IGS having undergone a phase transition due to its cooling because of the expansion of space. In contrast, the dark matter associated with the galaxies themselves did not develop such an index of refraction, since galaxies do not expand with the expansion of space, so that the speed of light within the galaxies remains the vacuum speed. Thus, in this model, dark energy is a different phase of dark matter, not a different substance, the particle nature of which is left open. For simplicity, it is left to later work to take into account that there will be a transition region at the outermost halo regions of galaxies, so that $n$ will not jump sharply from $n=1$ within a galaxy to $n \approx 1.5$ in the IGS. Also, since at $z=1.65 \pm 0.15$ the universe appears to have started to accelerate [19], it is assumed that the phase change into dark energy began at about this value of redshift, in which the index of refraction started to rise from $n=1$ to $n \approx 1.5$. Since the EdS universe continued to decelerate during this phase change there is no difficulty with the Co- 
pernican principle, as far as the expansion of space is concerned, unlike the case for the accelerating universe. However, at this point, there is a problem: The EdS universe works with cold dark matter, hence it is at zero temperature, yet the model assumes it was the cooling of dark matter in the IGS that led to the phase transition. The neglect of this problem is another simplification a more advanced model will have to address, possibly by working with some form of warm dark matter, for which there is wide discussion in the literature. Thus, in this highly simplified model, the dark energy contributes only to the energy density of the source tensor, so that with the dark matter plus baryon matter, for the flat EdS universe, one has $\Omega_{m}=1$. Although the flat EdS universe was used for comparison with the flat $\Lambda \mathrm{CDM}$ universe, the corrections provided by (1) or (2) do not require this. Thus in [7], the EdS value for $n(I s)$ was obtained for comparison with a closed universe with $\Omega_{m}=1.005$, a somewhat greater departure from unity than for Planck [20], with curvature density $\Omega_{k}= \pm 0.003$. There is also a critique of the cosmological term in conjunction with this study in [7], as well as another in [1].

\section{Electromagnetism and Density Parameters}

In an attempt to understand how the value of $n$ might be related to cosmological density parameters, it was noted in [6] that if one assumes the dark energy behaves as an isotropic linear medium in electromagnetic theory, then $n=\left(K K_{\mu}\right)^{1 / 2}$, where $K$ is the dielectric constant, and $K_{\mu}$ is the relative permeability, and that if one further assumes

$$
K K_{\mu}=\Omega_{d e} / \Omega_{m},
$$

where $\Omega_{d e}$ is the density parameter for dark energy, and is the same in magnitude as the density parameter associated with the cosmological term, so that $\Omega_{d e}=\Omega_{\Lambda}$, then

$$
n=\left(\Omega_{d e} / \Omega_{m}\right)^{1 / 2} .
$$

For the fiducial values of $\Omega_{m}$ and $\Omega_{\Lambda}$ used to determine $n$ from Equation (1), one obtains $n=1.53$, in good agreement with $n(l s)=1.49 \pm 0.04$. Since $n=1$ for $z>z^{\prime}$, where $z^{\prime}=1.65 \pm 0.15$, because the proposed phase change has not yet occurred, based on the finding in [19], Equation (4) only holds near the present epoch, where $n$ can be taken as constant. It was later found that if instead of basing $n(l s)$ on the range of redshifts $0.1 \leq z \leq 1.0$, one chose $0.1 \leq z \leq 0.6$, then $n(l s)=1.50 \pm 0.01$. This smaller range was chosen partly on the basis that the increasing disagreement with the $\Lambda \mathrm{CDM}$ at the higher redshifts is due to the possibility that the proposed phase transition is not complete, so that $n<1.5$ for these higher values of $z$, and also partly because kinematic considerations, given below, suggest $n=1.50$. This reduced range of redshifts includes the range for the BAO studies [17] [18] whose distance measurements were for galaxies with an effective redshift $z=0.57$. One can now use $n=1.50$ to re-determine $\Omega_{m}$ and $\Omega_{d e}$ by employing Equation (4), and the prediction 
that $\Omega_{m}+\Omega_{d e}=1$ from the EdS model, which is also the prediction of inflation [21] [22] [23], although independent of it. From these two relations one obtains

$$
\Omega_{m}=\left(1+n^{2}\right)^{-1}(0 \leq z \leq 0.6),
$$

and with $n^{2}=2.25 \pm 0.03$, one has $\Omega_{m}=0.308 \pm 0.003$, which is in perfect agreement with the Planck [20] value, $\Omega_{m}=0.308 \pm 0.012$, which in turn is in excellent agreement with the BOSS [24] value, $\Omega_{m}=0.310 \pm 0.005$, based on $\mathrm{BAO}$ studies of the flat $\Lambda \mathrm{CDM}$ universe. One can now redo the least squares determination of $n$, using the new values, $\Omega_{m}=0.308$, and $\Omega_{\Lambda}=\Omega_{d e}=0.692$, for the accelerating universe, and for $0.1 \leq z \leq 0.6$, and one finds again $n(l s)=1.50 \pm 0.01$. This value for $n$, together with further analysis, will make it possible next to resolve the problem concerning the age of the EdS universe. Note; Planck [25] gives slightly different values for $\Omega_{m}$ and the Hubble constant; however, in view of the magnitude of the uncertainties, such changes will be ignored.

Finally, to correct the discussion of the behavior of $\Omega_{d e}$ in [6], it was later noticed one should have $\Omega_{d e} \rightarrow 0$ for $z>\sim 1.7$ instead of $\Omega_{d e} \rightarrow \Omega_{m}$. This can be achieved if instead of (3) one sets

$$
\Omega_{d e} / \Omega_{m}=K K_{\mu} f^{2},
$$

so that $\left(\Omega_{d e} / \Omega_{m}\right)^{1 / 2}=n f$, where $f=f(z)$, and $f=1$ for $0 \leq z \leq 0.6$, and $f(z) \rightarrow 0$ for $z>\sim 1.7$. Also, $n=\sim 1.5$ for $0 \leq z \leq 0.6$, and $n \rightarrow 1$ for $z>\sim 1.7$. Then one has

$$
\Omega_{m}=\left(1+n^{2} f^{2}\right)^{-1}, \Omega_{d e}=n^{2} f^{2}\left(1+n^{2} f^{2}\right)^{-1},
$$

and hence for $z>\sim 1.7, \Omega_{m} \rightarrow 1$, and $\Omega_{d e} \rightarrow 0$, as required. It is possible the contemplated unified theory will help to justify (6).

\section{Age of the Universe, and the Hubble Constant}

As is well-known, the age problem arises as follows: The expansion parameter in the Friedmann, Le Maître Robertson Walker (FLRW) line element, $\mathrm{d} s^{2}=c^{2} \mathrm{~d} t^{2}-a(t)^{2}\left(\delta_{i j} \mathrm{~d} x^{i} \mathrm{~d} x^{j}\right),(i, j=1,2,3)$, for the flat EdS universe, obeys the equation $a(t)=a_{0}\left(t / t_{0}\right)^{2 / 3}$, where $t_{0}$ is the time from the Big Bang to the present epoch, for which $a\left(t_{0}\right)=a_{0}$, and hence it is the age of the EdS universe. The Hubble constant $H_{0}$, from its definition as $\dot{a}_{0} / a_{0}$, is therefore given by $H_{0}=2 / 3 t_{0}$, so that the age satisfies $t_{0}=(2 / 3) H_{0}^{-1}$. For the approximate value, $H_{0}=70 \mathrm{~km} \cdot \mathrm{s}^{-1} \cdot \mathrm{Mpc}^{-1}$ (current values of $H_{0}$ are given below), one has $t_{0}=9.3 \mathrm{Gyr}$, which is in significant disagreement with the ages of the oldest globular clusters in the MW, with a best fit of 12.6 Gyr [26]. The resolution is to notice that one of the assumptions that is made in determining the Hubble constant empirically is that the light from the red-shifted galaxies traveled through the dark energy of IGS with speed c. However, apart from the time light spent traveling through the MW with speed $c$ (which can be neglected for this preliminary work since it is estimated to contribute less than $\sim 0.5 \%$ ), according to the 
model, the light actually traveled through IGS with speed $c / n$. Also, since the redshifts used in determining $H_{0}$ satisfy $z<0.15$, as noted below, it is justifiable to treat $n$ as a constant. Then, from the first-order Doppler-effect, that can be used to determine $H_{0}$, when higher-order corrections are neglected, one has $\lambda_{0}=\lambda(1+n v / c)$, instead of $\lambda_{0}=\lambda(1+v / c)$, where $v$ is the speed of the receding galaxy after possible peculiar velocity has been taken into account.. Hence, with $z \equiv\left(\lambda_{0}-\lambda\right) / \lambda$, one has $v=c z / n$, instead of the customary $v=c z$. Since the relation that originally defined the Hubble constant can be written $H_{0} \equiv v / D=c z / D$, where $D$ is the proper distance to the receding galaxy, upon introducing the expression $H_{0}(c / n) \equiv c z / n D$, to allow for the reduced speed of light, one has

$$
H_{0}(c / n)=H_{0} / n \text {. }
$$

It follows that the age of the EdS universe, instead of that given above, is actually given by

$$
t_{0}=(2 / 3) H_{0}^{-1}(c / n)=(2 n / 3) H_{0}^{-1} .
$$

For $n=1.50$ there is obviously an exact cancellation of the $2 / 3$ factor (to be further discussed below), so that the revised age of the EdS universe is $t_{0}=H_{0}^{-1}$, which is the same as for a universe that had expanded uniformly. For $H_{0}=67.8 \pm 0.9 \mathrm{~km} \cdot \mathrm{s}^{-1} \cdot \mathrm{Mpc}^{-1}$, from Planck [20], and from (9), for $n=1.50$, the revised age of the EdS universe is

$$
t_{0}=H_{0}^{-1}=14.4 \pm 0.2 \mathrm{Gyr} .
$$

It will be noted that this age is significantly greater than the age of the $\Lambda \mathrm{CDM}$ universe given by Planck [20] as $13.8 \pm 0.1 \mathrm{Gyr}$. This is because the decelerating EdS universe necessarily takes longer to expand to $a_{0}$ than the accelerating universe. It was noted in [7] that the ages of the oldest stars in the MW could help to determine which age is more likely. For example, the subgiant HD140283 was found to have an age of $14.46 \pm 0.8 \mathrm{Gyr}$ [27] that is accommodated more readily by the EdS age than that of the accelerating universe. Nevertheless, as pointed out in [27], because of the large uncertainty in the star's age, it could still be younger than the age of the universe, given then as $13.77 \pm 0.06 \mathrm{Gyr}$. A related issue concerns the present tension between the Planck [20] value of $H_{0}$, based on the $\mathrm{CMB}$, and the value $H_{0}=73.24 \pm 1.74 \mathrm{~km} \cdot \mathrm{s}^{-1} \cdot \mathrm{Mpc}^{-1}$ [28], that emerges from a local determination, based on the cosmic distance ladder (CDL) that uses the Cepheid variables, and the SNe Ia for $z<0.15$. This tension shows up for the model in the following way: Thus, the CDL determination of $H_{0}$ leads to a Hubble time, $H_{0}^{-1}=13.4 \pm 0.3 \mathrm{Gyr}$, and hence with $n=1.50$ in (9), yields an age for the EdS universe of $13.4 \pm 0.3 \mathrm{Gyr}$, which is in tension with the age of HD140283. In contrast, the Planck [20] value of $H_{0}$ is consistent with a BAO determination that yielded $H_{0}=68.11 \pm 0.86 \mathrm{~km} \cdot \mathrm{s}^{-1} \cdot \mathrm{Mpc}^{-1}$ [29], and the value from BOSS that yielded, $H_{0}=67.6 \pm 0.5 \mathrm{~km} \cdot \mathrm{s}^{-1} \cdot \mathrm{Mpc}^{-1}$ [24]. However, HOLiCOW [30], based on gravitational lensing, employed a fiducial value $H_{0}=72 \mathrm{~km} \cdot \mathrm{s}^{-1} \cdot \mathrm{Mpc}^{-1}$, which favors the CDL value, hence HOLiCOW is also 
in tension with the $\mathrm{CMB}$ and BAO determinations, as discussed in the literature, and hence with the model as well. Also, as noted in [2], the GW signal, together with redshift measurement, yield $H_{0}=70_{-8}^{+12} \mathrm{~km} \cdot \mathrm{s}^{-1} \cdot \mathrm{Mpc}^{-1}$ [31]; however, because of the large uncertainty, it is compatible with both groups.

It was noted above that for $n=1.50$, there is an exact cancellation of the $2 / 3$ factor in (9), which would seem to imply that $n$ is the reciprocal of the $2 / 3$ factor. Although a dynamical explanation has not been found, there is the following kinematic explanation. Since the Hubble constant for a universe that expands uniformly, denoted here by $H_{0}(u n)$, satisfies $H_{0}(u n)=t_{0}^{-1}$, and since $H_{0}=2 / 3 t_{0}$ for the EdS universe, one has $H_{0}(\mathrm{EdS})=(2 / 3) H_{0}($ un $)$. Hence, in (8), if one sets $H_{0}(c / n)=H_{0}(\mathrm{EdS})$, and $H_{0}=H_{0}($ un $)$, one obtains $n=3 / 2$. However, this does not explain dynamically how the dark energy could acquire this value of $n$, along with the absence of dispersion. As suggested above, possibly such an explanation, including one for (6) would emerge from the contemplated unified theory, as well as a prediction of the functions $n(z)$ and $f(z)$.

\section{Absence of Neutrinos Correlated with GRBs}

Another type of prediction concerns failed attempts to correlate high energy neutrinos with gamma ray bursts (GRBs) as discussed in [6], which will be further extended here by considering a lower range of neutrino energies that was not discussed earlier. But before doing this, it is appropriate to comment on the neutrinos from SN 1987a [32] [33] [34] in the Large Magellanic Cloud. It was found that the light arrived 2 - $3 \mathrm{hrs}$ after the neutrino detection, see ref. 1 in [32]. However, if dark energy were present between the LMC and the MW, that extended for a distance as small as, say, one light-day, a reduced speed for that distance with $n \approx 1.5$ would have led to a time difference of $\sim 12 \mathrm{hrs}$, plus the time for the light to leave the supernova. Although a smaller distance cannot be ruled out, it is more likely the dark matter halo of the MW overlaps with the LMC in such a way that the cosmological expansion of space between the two galaxies either did not occur, or if it did, it was negligibly small. Thus, in this sense, the LMC is not extragalactic to the MW.

For brevity, only IceCube [12] [13] and Borexino [14] [15] findings will be discussed next, but other searches for a wide range of energies with the same null results are in [35]-[43]. As noted in [6], IceCube [12] found no correlation of high energy neutrinos with GRBs, while in [13] two PeV neutrinos were observed that were not correlated with any GRB. One possible explanation is that the sources of GRBs, although enormously energetic, do not produce a fluence of high energy neutrinos sufficient enough to be detected, and that the $\mathrm{PeV}$ neutrinos just happened to be passing through IceCube, is discussed in [13]. The proposed model suggests another possibility: Since according to it, GRBs travel with speed $\sim 2 c / 3$ through IGS, even if GRB sources did produce a sufficient fluence of neutrinos, they would have arrived millions of years before IceCube was built. While the two PeV neutrinos were possibly associated with an event that 
produced a GRB that will not arrive until the distant future. Indeed, according to the model, under the well-established prior that GRBs, along with accompanying optical and radio waves [44] [45] [46], arise from cosmologically-distant sources, neutrinos from such sources, even if the fluence were sufficient to exceed background, could never show any correlation with a GRB. This prediction is supported by Borexino's failure to find any low-energy (1.8 MeV - $17 \mathrm{MeV})$ neutrino and anti-neutrino signals correlated with GRBs [14]. In particular, Borexino's search from 2007 to 2015 for neutrino and anti-neutrino events correlated with 2350 GRBs, found no statistically significant excess over background. A possible explanation that assumes that there was too small a fluence of neutrinos and anti-neutrinos from GRB sources requires 2350 assumptions, one for each event, with no way to check empirically. More recently, Borexino searched for neutrinos correlated with the arrival of GWs [15], but none were found, beyond solar and background neutrinos. Thus, it is sufficient for the above proposition to be valid, if the speed of the GRBs and the GWs through IGS is $\sim 2 c / 3$ for $z \leq 0.6$. Indeed, from the standpoint of Occam's Razor, it is the preferable explanation, since it is much simpler. Two astronomical tests of this assumption for visible light are of special interest, because they can be readily carried out, and for completeness will be summarized next, with some abbreviations to the discussion in [8], as well as some improvements.

\section{Discordant Redshifts}

Astronomers have observed galaxies to have seemingly close companions with higher redshifts that have led to their description as "discordant redshifts". The current explanation, in accordance with the generally accepted view that higher redshifts correspond to greater distances, is that the higher redshift companion is the image of a galaxy that is behind the lower redshift galaxy, and shining through, thereby giving the impression that it is near the lower redshift galaxy, even though it is at an astronomically-large distance behind it. The term, "accidental superposition," is used to describe this scenario. An analysis of 64 cases of discordant redshifts, compiled by Arp [47], who disputed the standard explanation, led Bahcall [48], after making suitable assumptions, to estimate that $\sim 40$ cases were due to accidental superposition. He went on to conclude that, in view of the large uncertainty in the numbers he was using, all the cases were due to accidental superposition. However, the author's re-examination in [8] of Bahcall's estimate [48], found that he had rounded-up two of his proposed numbers before taking the product that led to the estimated $\sim 40$ cases, and that when one did not round-up, the $\sim 40$ cases were reduced to 30 cases. This suggested that about half of the 64 cases are due to some other cause, which the model provides, as will be shown next.

Assume that off to the side, angle-wise, of the lower redshift galaxy there is a higher redshift galaxy that can be viewed directly by the astronomer, although, because of the well-known general relativistic deflection of light that passes out- 
side, but near, the lower redshift galaxy, the higher redshift galaxy will appear shifted by a small angle from its actual position. Also, a light ray from the higher redshift galaxy, that travels at a suitable angle with respect to the direct ray, and strikes the lower redshift galaxy at an angle $\alpha$, less than the critical angle relative to the local normal, upon entering the galaxy, because of Snell's law, will be bent away from the normal by an angle $\beta$, with $\beta>\alpha$, in accordance with $n \approx 1.5$ for IGS, and $n=1$ for the galaxy, Since galaxies do not have sharp boundaries, the actual refraction will take place gradually, and depend on the variation of the index of refraction from its value in IGS, to that within the galaxy. However, if the variation is sufficiently smooth, this should not affect the qualitative result. After entry, the light will then travel straight through the lower redshift galaxy, in the absence of scattering and/or absorption, and, upon exiting, it will be bent towards the normal, and then, under suitable geometrical conditions, travel on to the astronomer, who will see an image of the higher redshift galaxy as a companion to the lower redshift galaxy, and describe it as a discordant redshift. An idealized figure is in [8] in which the lower redshift galaxy is treated as a perfect sphere with a sharp boundary, and refraction of the light entering the $\mathrm{MW}$ is ignored.

Thus, to test the model, astronomers might look for higher redshift galaxies that are off to the side, angle-wise, of lower redshift galaxies that match the higher redshift of the companions and their other features. If such galaxies were found, it would validate the model, but if they were not found, it would not necessarily falsify it, since such a finding would indicate that Bahcall's conclusion was correct, but not some of his estimated numbers. However, the model would certainly be falsified if a higher redshift galaxy were found off to the side, angle-wise, of a lower redshift galaxy, and in such a position that it could have given rise to a discordant redshift companion, but did not, since this would mean that light had entered the lower redshift galaxy without experiencing refraction, in conflict with the model.

\section{Conclusions}

If the model were to pass the proposed astronomical tests, not only would it provide an alternative explanation to the accelerating universe for the increased apparent magnitudes of the $\mathrm{SNe} \mathrm{Ia}$, and the increased distances to the standard ruler of the BAO, but in view of the recent discovery [1] [2] [3] [4] that the speed of a GW is the same as that of a GRB, it would indicate that GWs also experience the same reduction in their speed through the dark energy of IGS as EMWs, a possibility that was not considered in [5] [6] [7] [8]. This possibility, along with the proposed relation of EM parameters to gravitational parameters in (3) and (6), together with the absence of dispersion, may be seen as implications of Einstein's long-sought unified theory of gravitation and electromagnetism. Other predictions of the model are: dark energy as a different phase of dark matter, an increased age of the universe, a reduced value for the Hubble constant, and, significantly, the absence of the arrival of neutrinos correlated with either GRBs 
or GWs, a prediction that, in the case of GRBs, is already supported by numerous observations.

\section{References}

[1] Abbott, B.P., et al. (2017) LIGO Scientific Collaboration and Virgo Collaboration: Astrophysical Journal Letters, 848, L12. https://doi.org/10.3847/2041-8213/aa91c9

[2] Abbott, B.P., et al. (2017) LIGO Scientific Collaboration and Virgo Collaboration: Physical Review Letters, 119, 161101. https://doi.org/10.1103/PhysRevLett.119.161101

[3] Goldstein, A., et al. (2017) Fermi-GBM, Astrophysical Journal Letters, 848, L14. https://doi.org/10.3847/2041-8213/aa8f41

[4] Savchenko, V., et al. (2017) INTEGRAL Collaboration, Astrophysical Journal Letters, 848, L15. https://doi.org/10.3847/2041-8213/aa8f94

[5] Tangherlini, F.R. (2015) Journal Modern Physics, 6, 78-87. https://doi.org/10.4236/jmp.2015.61010

[6] Tangherlini, F.R. (2015) Journal Modern Physics, 6, 1360-1370. https://doi.org/10.4236/jmp.2015.69141

[7] Tangherlini, F.R. (2016) Journal Modern Physics, 7, 1829-1844. https://doi.org/10.4236/jmp.2016.713163

[8] Tangherlini, F.R. (2017) Journal Modern Physics, 8, 622-635. https://doi.org/10.4236/jmp.2017.84042

[9] Riess, A.G., et al. (1998) Astronomical Journal, 116, 1009-1038. https://doi.org/10.1086/300499

[10] Schmidt, B., et al. (1998) Astrophysical Journal, 507, 45-63. https://doi.org/10.1086/306308

[11] Perlmutter, S., et al. (1999) Astrophysical Journal, 517, 565-586. https://doi.org/10.1086/307221

[12] Abbasi, R., et al. (2012) IceCube Collaboration: Nature, 484, 351-354. https://doi.org/10.1038/nature11068

[13] Artsen, M.G., et al. (2013) IceCube Collaboration: Physical Review Letters, 111, Article ID: 021103.

[14] Agostini, M., et al. (2017) Borexino Collaboration, Astroparticle Physics, 86, 1-17.

[15] Agostini, M., et al. (2017) Borexino Collaboration, Astrophysical Journal, 850, 21.

[16] Tonry, J.L., et al. (2003) Astrophysical Journal, 594, 1-24. https://doi.org/10.1086/376865

[17] Anderson, L., et al. (2012) Monthly Notices Royal Astronomical Society, 427, 3435-3457. https://doi.org/10.1111/j.1365-2966.2012.22066.x

[18] Anderson, L., et al. (2014) Monthly Notices Royal Astronomical Society, 439, 83-101. https://doi.org/10.1093/mnras/stt2206

[19] Riess, A.G., et al. (2001) Astrophysical Journal, 560, 49-71. https://doi.org/10.1086/322348

[20] Abe, P.A.R., et al. (2015) Planck Collaboration: Astronomy and Astrophysics, 584, $1-67$.

[21] Guth, A.H. (1981) Physical Review D, 23, 347-356. https://doi.org/10.1103/PhysRevD.23.347

[22] Linde, A.D. (1982) Physics Letters B, 108, 389-392. https://doi.org/10.1016/0370-2693(82)91219-9 
[23] Albrecht, A. and Steinhardt, P. (1982) Physical Review Letters, 48, 1220-1223. https://doi.org/10.1103/PhysRevLett.48.1220

[24] Shadab, A., et al. (2016) BOSS Collaboration, Monthly Notices Royal Astronomical Society, 000, 1-38.

[25] Abe, P.A.R., et al. (2016) Planck Collaboration: Astronomy and Astrophysics, 594, A13.

[26] Krauss, L.M. and Chaboyer, B. (2003) Science, 299, 65-69. https://doi.org/10.1126/science.1075631

[27] Bond, H.E., Nelan, E.P., VandenBerg, D.A., Schaefer, G.H. and Harmer, D. (2013) Astrophysical Journal Letters, 765, L12. https://doi.org/10.1088/2041-8205/765/1/L12

[28] Riess, A.G., et al. (2016) Astrophysical Journal, 826, 56.

[29] Chang, C. and Huang, Q. (2015) Science China Physics, Mechanics and Astronomy, 58, Article ID: 599801. https://doi.org/10.1007/s11433-015-5684-5

[30] Suyu, S.H., et al. (2017) HOLiCOW 1, Monthly Notices Royal Astronomical Society, 000, 1-15.

[31] Abbott, B.P., et al. (2017) Nature (London), 551, 85-88.

[32] Hirata, K., et al. (1987) Physical Review Letters, 58, 1490-1493. https://doi.org/10.1103/PhysRevLett.58.1490

[33] Bionta, R.M., et al. (1987) Physical Review Letters, 58, 1494-1496. https://doi.org/10.1103/PhysRevLett.58.1494

[34] Alekseev, E.N., et al. (1987) JETP Letters, 45, 589-592.

[35] Achteberg, A., et al. (2007) Astrophysical Journal, 664, 397-410.

[36] Vieregg, A.G., et al. (2011) Astrophysical Journal, 736, 50.

[37] Adrián-Martínez, S., et al. (2017) European Physical Journal C, 77, 20.

[38] Adrián-Martínez, S., et al. (2013) Astronomy \& Astrophysics, 559, A9. https://doi.org/10.1051/0004-6361/201322169

[39] Avrorin, A., et al. (2011) Physics of Particles and Nuclei Letters, 8, 704-716. https://doi.org/10.1134/S154747711107003X

[40] Fukuda, S., et al. (2002) Astrophysical Journal, 578, 317-324. https://doi.org/10.1086/342405

[41] Aharmim, B., et al. (2014) Astroparticle Physics, 55, 1-76. https://doi.org/10.1016/j.astropartphys.2013.12.004

[42] Asakura, K., et al. (2015) Astrophysical Journal, 806, 87.

[43] Kochkarov, M.M., et al. (2015) Physics of Particles and Nuclei, 46, 197. https://doi.org/10.1134/S1063779615020124

[44] Costa, E., et al. (1997) Nature (London), 387, 783-785. https://doi.org/10.1038/42885

[45] Metzger, M.R., et al. (1997) Nature (London), 387, 878-880. https://doi.org/10.1038/43132

[46] Frail, D.A., et al. (1997) Nature (London), 389, 261-263. https://doi.org/10.1038/38451

[47] Arp, H. (1996) Astrophysical Journal Supplement, 14, 1-20.

[48] Bahcall, J.N. (1976) Redshifts as Distance Indicators. In: Field, G.B., Bahcall, J.N. and Arp, H., Eds., The Redshift Controversy, W. A. Benjamin Inc., Reading, 61-121. 